\title{
Fitting the Modified-Power-Lognormal to the Sum of Independent Lognormals Distribution
}

\author{
Sebastian S. Szyszkowicz and Halim Yanikomeroglu \\ Dept. of Systems and Computer Engineering \\ Carleton University \\ Ottawa, Ontario, Canada \\ E-mail: \{sz, halim\}@sce.carleton.ca
}

\begin{abstract}
We propose a new method for calculating a tight approximation to the distribution of the sum of independent lognormal random variables. We make use of a three-parameter modified-power-lognormal distribution function as the approximating distribution. We use theoretical results from our previous work on the tails of the distribution of the sum of lognormals to match the slope of the modified-power-lognormal function at both tails. This would not have been possible with many of the recently-proposed distribution functions, which do not behave properly in the tails. We then also use moment-matching to find the best curve match. Our method is mostly closed-form, requiring only one simple numerical integral.

We compare our method with those in literature in terms of complexity and accuracy. We conclude that our method is more accurate than the simple (closed-form) methods, and much simpler to understand and implement than the more accurate methods which rely heavily on numerical integration.

Index Terms-sum of lognormals, interference analysis
\end{abstract}

\section{INTRODUCTION}

The mathematical problem of the sum of lognormals (SLN) arises in wireless communications, in particular when one analyses the total power received from several interfering sources [1]-[3]. It has been the object of many papers, also in other scientific fields [4]-[8].

The problem can be mathematically stated as follows: let $\left\{Y_{i}\right\}_{i=1}^{N}$ be a set of lognormal random variables such that

$$
\begin{gathered}
Y_{i} \sim f_{i}\left(x ; \mu_{i}, \sigma_{i}\right)=\frac{1}{\sqrt{2 \pi} x \sigma_{i}} e^{-\frac{1}{2}\left(\frac{\ln x-\mu_{i}}{\sigma_{i}}\right)^{2}} \\
=\frac{\partial}{\partial x} F_{i}\left(x ; \mu_{i}, \sigma_{i}\right)=\frac{\partial}{\partial x} \Phi\left(\frac{\ln x-\mu_{i}}{\sigma_{i}}\right), x>0,
\end{gathered}
$$

where $\Phi(x)$ is the standard normal cumulative distribution function $(c d f)$. We have, for any real number $n$ [9]:

$$
\mathbb{E}\left\{Y_{i}^{n}\right\}=e^{n \mu_{i}+\frac{1}{2} n^{2} \sigma_{i}^{2}}
$$

We then say that

$$
X=\sum_{i=1}^{N} Y_{i} \sim f(x)=\frac{\partial}{\partial x} F(x), x>0
$$

This work was supported in part by a $P G S D$ award from the National Sciences and Engineering Research Council (NSERC) of Canada, and by Wireless Technology Labs, Nortel Networks, Ottawa, Canada. follows the SLN distribution, which has no known closed form. When the summands $Y_{i}$ are independent, $f(x)$ is found by convolution of the marginal densities:

$$
f(x)=f_{1}(x) \otimes f_{2}(x) \otimes \cdots \otimes f_{N}(x) .
$$

An exact closed-form method for performing even one of these convolutions is not known.

There exist, however, many approximate methods for finding the SLN distribution [9]-[24]. These methods can be categorised by their scope into three classes: generally correlated [9]-[15], independent [16]-[21], independent and identically distributed [22]-[24] $Y_{i}$ 's. We can also categorise the methods based on their complexity: most methods require extensive numerical integration, and many are iterative. Only [9], [10], [16] are closed-form, and [18] is mostly closed-form, similarly to our method.

In this paper we analyse the sum of independent lognormal random variables with arbitrary parameters. The input to the problem is thus the set of pairs $\left\{\left(\mu_{i}, \sigma_{i}^{2}\right)\right\}_{i=1}^{N}$. The goal of our work is to develop a distribution function that can approximate the SLN distribution well over the entire range of values. The approximating distribution function should be explicitly described by only a few parameters, which need to be found for each set of input parameters according to a given algorithm, which should be simple. Thus any parametric method for solving the SLN problem consists of two parts: finding an adequate analytical form for the distribution function, and evaluating that function's parameters for each particular case.

We use the 3-parameter Modified-Power-Lognormal (MPLN) distribution as our approximating function, which has been previously proposed for approximating the SLN $c d f$ in various ways [3], [5], [13], [16], [22]. In Section II, we summarise the many arguments, some novel, for the choice of this particular distribution. In Section III, we introduce a simple new, mostly closed-form method for obtaining the three MPLN parameters a priori (without prior knowledge of the SLN $c d f$ ). In Section IV, we compare our method against other closed-form methods, as well as against MonteCarlo simulations and argue that our method is of practical use, because it outperforms the existing closed-form methods, and is much simpler than all existing numerical methods. We conclude in Section V. 


\section{Choice of The MPLN Distribution}

\section{A. A Note on Nomenclature}

To the best of our knowledge, the distribution given by

$$
\begin{aligned}
& f_{\mathrm{PLN}}(x)=\frac{t}{\sqrt{2 \pi} x s} e^{-\frac{1}{2}\left(\frac{\ln x-m}{s}\right)^{2}} \Phi^{t-1}\left(\frac{m-\ln x}{s}\right), \\
& F_{\mathrm{PLN}}(x)=1-\Phi^{t}\left(\frac{m-\ln x}{s}\right), x \geq 0, s>0, t>0
\end{aligned}
$$

has always been called the power-lognormal (PLN) distribution [25], [26]. The distribution we use to approximate the SLN will be

$$
\begin{aligned}
& f_{\mathrm{MPLN}}(x)=\frac{t}{\sqrt{2 \pi} x s} e^{-\frac{1}{2}\left(\frac{\ln x-m}{s}\right)^{2}} \Phi^{t-1}\left(\frac{\ln x-m}{s}\right), \\
& F_{\text {MPLN }}(x)=\Phi^{t}\left(\frac{\ln x-m}{s}\right), x \geq 0, s>0, t>0 .
\end{aligned}
$$

We refer to this distribution as the modified-power-lognormal (MPLN) distribution, though it has been inconsistently called PLN in the past both by us [3] and in [13]. It could be argued that the name "power-lognormal" is more natural for the distribution in (6) than the one in (5). However, we decided on the current nomenclature for consistency with the majority of past literature.

\section{B. Historical Motivation}

This function has been proposed by Farley [22] for analysing the SLN distribution in the independent and identically distributed case. We then have $s=\sigma=\sigma_{1}=\sigma_{2}=$ $\ldots=\sigma_{N}, m=\mu=\mu_{1}=\mu_{2}=\ldots=\mu_{N}, t=N$. In this case, our method essentially reduces to Farley's method for finding $s$ (12), and $t$ (13), but $m$ is found differently (17) so as to match the first moment of $X$. Our method simply shifts the curve a little downwards to balance out the error in the different parts of the $c d f$.

Farley's method was extended by Ben Slimane [16] to include non-identical independent summands. Like Farley's, it is based on the fact that the sum of positive random variables is always greater or equal than the greatest of these random variables. For lognormal random variables, which have very large spreads, this approximation tends to be quite good, and is tight in the upper tail (as $x \rightarrow+\infty$ ). This leads to the bound (which is also often a good approximation)

$$
X \gtrsim \max _{i} Y_{i} \Rightarrow F(x) \lesssim \prod_{i=1}^{N} F_{i}(x) .
$$

The difficulty with this method is that it is non-parametric: in general, in needs to be expressed as a function of $2 N$ parameters and therefore does not lead to simple subsequent analytical treatment.

Our method can be considered a synthesis of both Farley's and Ben Slimane's approximations. We only use 3 parameters, but we consider the sum of any independent lognormal distributions.

This choice of the MPLN form is also motivated by [5], which suggests that the SLN distribution could be expressed as a sum of products of lognormal distributions, of which the MPLN distribution is a particular case.

The MPLN distribution was proposed again in [13] for approximating the SLN distribution, including the correlated case. However, it gave an a posteriori method for finding the MPLN parameters, by fitting the MPLN $c d f$ to a pre-computed SLN $c d f$. Obtaining the exact $c d f$ requires lengthy MonteCarlo simulations, or advanced slowly-converging numerical methods [19], and then a numerical solver is also required for the curve-fitting, making the method in [13] complex and numerically intensive.

\section{Working on Lognormal Paper}

It is convenient to analyse some $c d f$ 's on lognormal paper [19], i.e., under the transformation $T$

$$
T: F(x) \mapsto \Phi^{-1}\left(F\left(e^{y}\right)\right) .
$$

Under this transformation, each lognormal distribution is mapped onto a linear equation with positive slope, and vice versa. Also, when plotted on lognormal paper, the SLN distribution has linear asymptotes: certainly in the $+\infty$ tail, and probably in the $-\infty$ tail as well [27].

We thus consider the MPLN $c d f$ on lognormal paper:

$$
T: F_{\mathrm{MPLN}}(x) \mapsto \tilde{F}_{\mathrm{MPLN}}(y),
$$

where $T$ is defined in (8).

\section{Tail Properties on Lognormal Paper}

We proved in [27] that, when plotted on lognormal paper, the independent SLN distribution has linear asymptotes with simple closed-form expressions: certainly in the $+\infty$ tail, and probably (we could not prove it formally) in the $-\infty$ tail as well. It is therefore interesting to use an approximating function that also has linear asymptotes in its tails.

Thus, we also chose the MPLN distribution for its tail behaviour. Under the transformation $T$, the MPLN distribution has a linear asymptote in the upper limit with slope

$$
\lim _{y \rightarrow+\infty} \frac{\partial}{\partial y} \tilde{F}_{\mathrm{MPLN}}(y)=\frac{1}{s},
$$

In the lower limit, it has no linear asymptote, but does have a limiting slope

$$
\lim _{y \rightarrow-\infty} \frac{\partial}{\partial y} \tilde{F}_{\mathrm{MPLN}}(y)=\frac{\sqrt{t}}{s} .
$$

These results are proved in Appendix A. Therefore, it will be easy to match the tail slopes of the MPLN with those of the SLN distribution to solve for the MPLN parameters.

This would not be possible with many new proposed distributions [11], [21], [23], [24], which although they may approximate the SLN distribution very well, were showed in [27] not to have linear asymptotes on lognormal paper at either tail. Therefore the closed-form results from [27] on the SLN tails cannot be exploited. Two recent papers [17], [18] proposed new distribution functions. We show in Appendices B and C why we cannot apply tail-matching for those distributions either. 
In general, we propose that this approach be used to solve the SLN problem in closed-form: find a distribution with lognormal tails, and fit them to the tails of the SLN distribution [4], [27]. So far, we were the only ones to take this approach, and only with the MPLN distribution, but perhaps other (better) distribution functions exist that are amenable to this approach.

\section{Parameter Matching}

We successively match the $s, t$, and $m$ parameters in that order. The first two parameters are found in closed form, and only the $m$ parameter requires some simple numerical integration.

\section{A. Upper Tail Slope}

We first obtain the $s$ parameter. The slope in the $+\infty$ tail of the SLN $c d f$ on lognormal paper is known to be $1 / \max _{i} \sigma_{i}$ [4], [27]. We equate this slope with (10):

$$
\frac{1}{\max _{i} \sigma_{i}}=\frac{1}{s} \Rightarrow s=\max _{i} \sigma_{i}
$$

\section{B. Lower Tail Slope}

Now, having the $s$ parameter, we find $t$. In [27] we argued that the slope in the $-\infty$ tail of the SLN distribution (for independent summands) is $\sqrt{\sum_{i=1}^{N} \sigma_{i}^{-2}}$. We match this slope with that of the MPLN distribution, given in (11):

$$
\frac{\sqrt{t}}{s}=\sqrt{\sum_{i=1}^{N} \sigma_{i}^{-2}} \Rightarrow t=s^{2} \sum_{i=1}^{N} \sigma_{i}^{-2} .
$$

In the special case of $\sigma_{1}=\sigma_{2}=\ldots=\sigma_{N}=\sigma$, we obtain simply $s=\sigma$ and $t=N$.

\section{Moment-Matching}

Having obtained $s$ and $t$, we may next find $m$. However, this requires a numerical integral,

$$
\Lambda(s, t)=\int_{-\infty}^{+\infty} e^{s x-\frac{1}{2} x^{2}} \Phi^{t-1}(x) d x,
$$

which is well approximated by a Riemann sum. This is substantially simpler to understand and implement than other numerical methods [11], [12], [17], [19]-[21], [23], [24]. The first moment of the MPLN distribution can be written as

$$
\int_{0}^{+\infty} x f_{\mathrm{MPLN}}(x) d x=t e^{m}(2 \pi)^{-\frac{1}{2}} \Lambda(s, t) .
$$

The first moment of the SLN can be found directly,

$$
\mathbb{E}\{X\}=\sum_{i=1}^{N} \mathbb{E}\left\{Y_{i}\right\}=\sum_{i=1}^{N} e^{\mu_{i}+\frac{1}{2} \sigma_{i}^{2}} .
$$

Equating (15) with (16) we obtain

$$
m=\ln \sum_{i=1}^{N} e^{\mu_{i}+\frac{1}{2} \sigma_{i}^{2}}-\ln \Lambda(s, t)-\ln t+\frac{1}{2} \ln 2 \pi .
$$

Thus our method can be summarised by the following steps: obtain $s$ from (12), $t$ from (13) and $m$ from (14) and (17), then substitute these into the distribution function (6).

\section{Closed-Form Case}

The integral (14) is of course solvable for $t=1$. It is also separable for $t=2$, which corresponds to the case where there are only two summands, with $\sigma_{1}=\sigma_{2}=s=\sigma$. The integral then has solution

$$
\Lambda(s, 2)=\int_{-\infty}^{+\infty} e^{s x-\frac{1}{2} x^{2}} \Phi(x) d x=\sqrt{2 \pi} \Phi\left(\frac{s}{\sqrt{2}}\right) e^{\frac{1}{2} s^{2}}
$$

which can be solved by rewriting $\Phi(x)$ as an integral, and performing a change of variables. This integral is known: it is the same as the moment-generating function of the skewnormal distribution [28], when the skew parameter is 1 .

Then $m$ can be found in closed form:

$$
m=\ln \left(e^{\mu_{1}}+e^{\mu_{2}}\right)-\ln 2-\ln \Phi\left(\frac{\sigma}{\sqrt{2}}\right) .
$$

And, if both summands have identical distribution, that is $\mu_{1}=$ $\mu_{2}=\mu$, then

$$
m=\mu-\ln \Phi\left(\frac{\sigma}{\sqrt{2}}\right) .
$$

Solving (14) for higher (and non-integer) values of $t$ remains an open problem.

\section{Simulations And COMParisons}

In Figures 1-3, we present Monte-Carlo simulations of the SLN $c d f$, along with three methods for approximating it: the proposed MPLN method, moment-matching [2], [9], [29] (using the first and second moments), and Ben Slimane's method (7) [16]. In the context of inter-cell co-channel interference, typical values of $\sigma_{i}$ are between 6 and $12 \mathrm{~dB}$. The equivalent $\sigma_{i}$ in natural units is found according to $\sigma_{i}[$ nat $]=\sigma_{i}[\mathrm{~dB}] \times 0.1 \ln 10$. We do not show the method in [10], which performs very poorly for values of $\sigma_{i}$ of more than $4 \mathrm{~dB}$, and is designed to analyse intra-cell interference in CDMA systems. We make the following observations:

1) In the case of identically distributed terms (Figure 1), the MPLN method is nearly identical to the bound method (which is equivalent to Farley's method in this case). Both these methods outperform moment-matching, particularly in the lower tail.

2) In the case of different $\mu_{i}$ 's (Figures 2 and 3), the MPLN method usually outperforms the other methods.

3) A few tests with widely different $\sigma_{i}$ 's (not shown here) did not yield good performance in any of the three methods. This is not a very bad thing in the wireless communications context, since shadowing spread is usually constant within a particular situation, while it is only the average gains that will significantly differ.

Now there exist several numerical methods that could perform better than our MPLN method in many cases. We should, however, make the following comments:

1) Several numerical methods approximate the SLN by another lognormal [1], [12], [19]. From Figures 1-3 and from literature, it is becoming evident that in many cases of interest this cannot possibly be accurate, since 


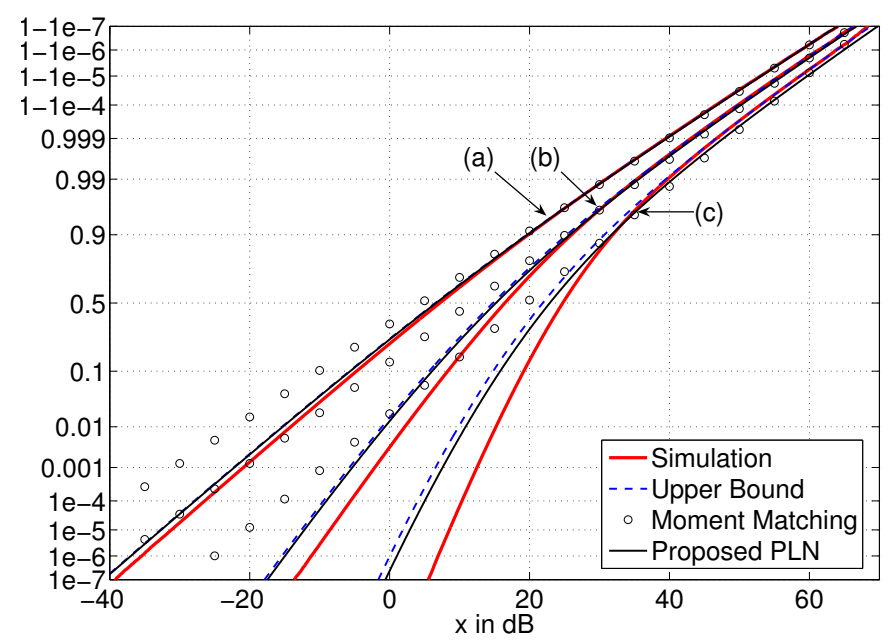

Fig. 1. Sum of independent identically distributed lognormals with $\mu_{i}=0$, $\sigma_{i}=12 \mathrm{~dB}=2.76$ nat and (a) $N=2$, (b) $N=6$, (c) $N=20$.

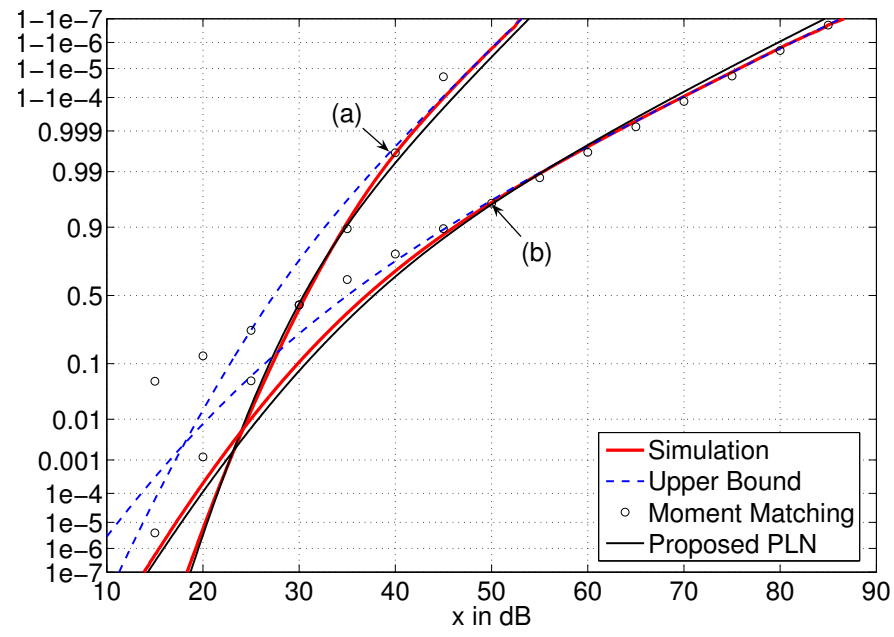

Fig. 2. Sum of independent lognormals with $N=20, \mu_{1}=\mu_{2}=\ldots=$ $\mu_{6}=20 \mathrm{~dB}=4.61$ nat, $\mu_{7}=\mu_{8}=\ldots=\mu_{20}=0$ and (a) $\sigma_{i}=6 \mathrm{~dB}=$ 1.38 nat, (b) $\sigma_{i}=12 \mathrm{~dB}=2.76$ nat.

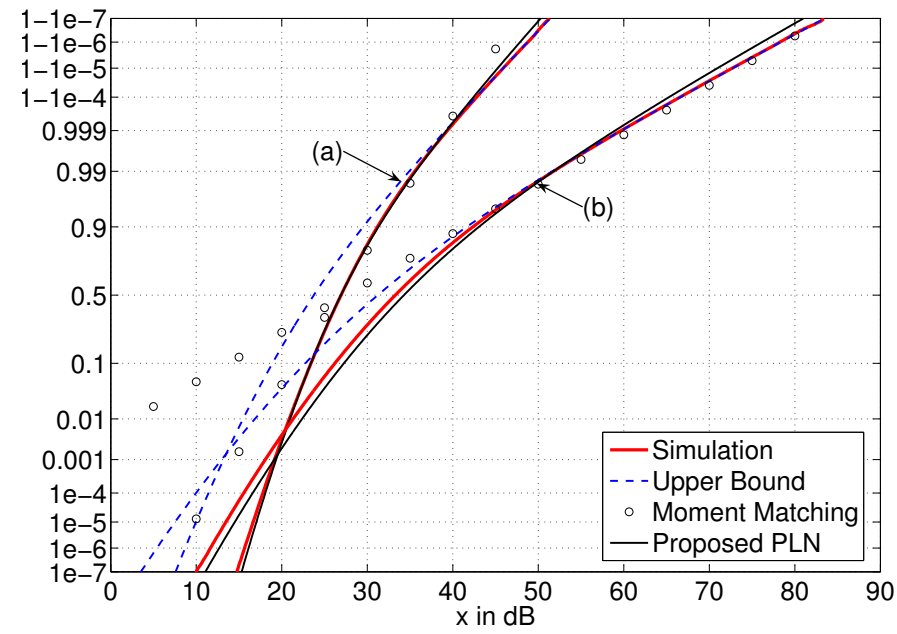

Fig. 3. Sum of independent lognormals with $N=21, \mu_{1}=-20 \mathrm{~dB}, \mu_{2}=$ $-18 \mathrm{~dB}, \mu_{3}=-16 \mathrm{~dB}, \ldots, \mu_{21}=20 \mathrm{~dB}$ and (a) $\sigma_{i}=6 \mathrm{~dB}=1.38$ nat, (b) $\sigma_{i}=12 \mathrm{~dB}=2.76$ nat. the SLN distribution is very non-linear on lognormal paper. This has lead to the recent proposal of several new approximating functions [11], [17], [21], [23], [24] and also to a piecewise-lognormal solution [20].

2) Most numerical SLN methods proposed involve iteration and complex numerical techniques [1], [11], [17], [19][21]. While the execution of these algorithms on a computer may be quasi-instantaneous, they still require significant human investment to understand and implement the method. Method [12] is perhaps not very complicated. However our method is much simpler to understand and can be implemented quickly with only a simple Riemann sum.

3) Some methods [23], [24] apply to sums of identically distributed lognormals only, and are essentially tabulations of function parameters for a necessarily limited set of cases.

Lastly, we must examine the method in [18]. It is very comparable to ours, in that it is closed-form apart from a simple numerical integration, and that it applies to any independent summands. Unfortunately, we did not have the time to implement their method to compare their exactness. However, there are a few qualitative factors that favour our method over theirs:

1) The lower tail of their method necessarily diverges from the true solution because the distribution has some mass at negative values, while $X$ is by definition positive (see Appendix C).

2) The solution might not exist for some combinations of parameters (specifically, low $N$ with high $\sigma_{i}[18]$ ).

3) Their $c d f$ is not known in closed form.

4) Our equations for identifying the parameters are still many times shorter than theirs.

\section{CONCLUSION}

The MPLN method introduced here seems to perform no worse, and often better, than the existing closed-form methods [9], [10], [16], while being of comparable complexity. Its performance is particularly good for cases where the $\sigma_{i}^{2}$ 's are all the same while the $\mu_{i}$ 's differ. This case is particularly interesting in wireless cellular communications, where both strong and weak interferers suffer shadowing with constant logarithmic variance. Our method is significantly simpler than the available numerical techniques, and if the integral (14) could be solved exactly or well approximated, our method would be entirely closed-form. 


\section{APPENDIX A}

We begin by showing that the MPLN distribution has a best lognormal fit [27] at $+\infty$. We use l'Hospital's rule to find

$$
\begin{aligned}
& \lim _{x \rightarrow+\infty} \frac{1-F_{\mathrm{MPLN}}(x)}{1-\Phi\left(\frac{\ln x-m}{s}\right)} \\
= & \lim _{x \rightarrow+\infty} \frac{f_{\mathrm{MPLN}}(x)}{\frac{1}{\sqrt{2 \pi} x s} \exp \left(-\frac{1}{2}\left(\frac{\ln x-m}{s}\right)^{2}\right)} \\
= & \lim _{x \rightarrow+\infty} t \Phi^{t-1}\left(\frac{\ln x-m}{s}\right)=t .
\end{aligned}
$$

Because this ratio converges to a finite non-zero value, we may conclude from [27, Lemma 2] that $\Phi\left(\frac{\ln x-m}{s}\right)$ is a best lognormal fit to $F_{\mathrm{MPLN}}(x)$ at $+\infty$. The corresponding slope is $\frac{1}{s}$ on lognormal paper, which proves (10).

In order to prove the lower tail slope, we first need a couple of approximations. A well-known approximation for $\Phi(x)$ is

$$
\Phi(x) \approx \frac{1}{-\sqrt{2 \pi} x} e^{-\frac{1}{2} x^{2}}, x \rightarrow-\infty .
$$

We also use an approximation from [30], after some manipulation:

$$
\Phi^{-1}(x) \approx-\sqrt{-2 \ln (2 x)-\ln (-\pi \ln (2 x))}, x \rightarrow 0^{+} .
$$

We may then write

$$
\begin{aligned}
& \lim _{y \rightarrow-\infty} \frac{\partial}{\partial y} \tilde{F}_{\mathrm{MPLN}}(y)=\frac{1}{s} \lim _{y \rightarrow-\infty} \frac{\partial}{\partial y} \Phi^{-1}\left(\Phi^{t}(y)\right) \\
= & -\frac{1}{s} \lim _{y \rightarrow-\infty} \frac{\partial}{\partial y} \sqrt{t y^{2}+\mathcal{O}(\ln (-y))} \\
= & -\frac{1}{s} \lim _{y \rightarrow-\infty} \frac{2 t y}{2 \sqrt{t y^{2}}}=\frac{\sqrt{t}}{s} .
\end{aligned}
$$

This proves (11).

\section{APPENDIX B}

In [17], a mixture of two lognormal distributions (M2LN) was proposed for approximating the SLN distribution

$$
F_{\mathrm{M} 2 \mathrm{LN}}(x)=\gamma \Phi\left(\frac{\ln x-\mu_{1}}{\sigma_{1}}\right)+(1-\gamma) \Phi\left(\frac{\ln x-\mu_{2}}{\sigma_{2}}\right),
$$

with $x>0$ and five parameters $0 \leq \gamma \leq 1, \mu_{1}, \sigma_{1}>$ $0, \mu_{2}, \sigma_{2}>0$.

We will prove that this distribution has a linear asymptote in both tails on lognormal paper. However, both asymptotes have the same slope, and thus this distribution cannot be matched to the asymptotic results in [27]. This is evident for $\gamma=0,1$ because the M2LN distribution then reduces to a simple lognormal. To prove this for $0<\gamma<1$, let us take an arbitrary lognormal distribution with parameters $\mu, \sigma^{2}$ and show that it can be an asymptote to the M2LN distribution on lognormal paper. For the upper tail, it is sufficient [27] to show that the following quantity converges to a finite non-zero value:

$$
\begin{aligned}
L_{+} & =\lim _{x \rightarrow+\infty} \frac{1-F_{\mathrm{M} 2 \mathrm{LN}}(x)}{1-\Phi\left(\frac{\ln x-\mu}{\sigma}\right)} \\
& =\lim _{x \rightarrow+\infty} \gamma \frac{\sigma}{\sigma_{1}} e^{\frac{1}{2}\left(\sigma^{-2}-\sigma_{1}^{-2}\right) x^{2}+\left(\mu_{1} \sigma_{1}^{-2}-\mu \sigma^{-2}\right) x+\mathcal{O}(1)} \\
& +(1-\gamma) \frac{\sigma}{\sigma_{2}} e^{\frac{1}{2}\left(\sigma^{-2}-\sigma_{2}^{-2}\right) x^{2}+\left(\mu_{2} \sigma_{2}^{-2}-\mu \sigma^{-2}\right) x+\mathcal{O}(1)} .
\end{aligned}
$$

From this we see that a necessary condition for $L_{+} \in(0,+\infty)$ is $\sigma=\sigma_{+}=\max \left(\sigma_{1}, \sigma_{2}\right)$, and hence, from [27, Lemma 2], it follows that the M2LN distribution has a linear asymptote at $+\infty$ on lognormal paper corresponding to a lognormal distribution with $\log$-variance $\sigma_{+}^{2}$.

Likewise, for the lower tail [27]

$$
\begin{aligned}
L_{-} & =\lim _{x \rightarrow-\infty} \frac{F_{\mathrm{M} 2 \mathrm{LN}}(x)}{\Phi\left(\frac{\ln x-\mu}{\sigma}\right)} \\
& =\lim _{x \rightarrow-\infty} \gamma \frac{\sigma}{\sigma_{1}} e^{\frac{1}{2}\left(\sigma^{-2}-\sigma_{1}^{-2}\right) x^{2}+\left(\mu_{1} \sigma_{1}^{-2}-\mu \sigma^{-2}\right) x+\mathcal{O}(1)} \\
& +(1-\gamma) \frac{\sigma}{\sigma_{2}} e^{\frac{1}{2}\left(\sigma^{-2}-\sigma_{2}^{-2}\right) x^{2}+\left(\mu_{2} \sigma_{2}^{-2}-\mu \sigma^{-2}\right) x+\mathcal{O}(1)} .
\end{aligned}
$$

Again we see that a necessary condition for $L_{-} \in(0,+\infty)$ is $\sigma=\sigma_{-}=\max \left(\sigma_{1}, \sigma_{2}\right)$, and hence, from [27, Lemma 2], it follows that the M2LN distribution has a linear asymptote at $-\infty$ on lognormal paper corresponding to a lognormal distribution with $\log$-variance $\sigma_{-}^{2}$.

Now $\sigma_{+}=\sigma_{-}$while the slopes at both tails of the SLN distribution on lognormal paper [27] for $N>1$ are different, and thus the M2LN distribution is not adequate for tailmatching.

\section{APPENDIX C}

In [18], a Pearson IV is proposed as an SLN approximation, with $p d f$

$$
f_{\mathrm{ZP}}(x)=\nu\left(1+\left(\frac{x-\mu_{4}}{\mu_{3}}\right)^{2}\right)^{-\mu_{1}} e^{-\mu_{2} \arctan \left(\frac{x-\mu_{4}}{\mu_{3}}\right)},
$$

with $x \in \mathbb{R}$ and no general closed form for the $c d f$. Note that the $p d f$ will not be valid unless $\mu_{1}>\frac{1}{2}$.

To analyse the tails of this distribution, we first notice that the distribution does not stop at $x=0$, but can take negative values. For this reason, it is impossible to match the lower tail with that of an SLN, which forcibly takes positive values only. This is seen in the figures of [18], where the lower tails totally diverge. The same symptom is observed in the distribution of [11].

As for the upper tail, the analysis is as follows:

$$
f_{\mathrm{ZP}}(x) \approx \nu \mu_{3}^{2 \mu_{1}} e^{-\frac{\pi}{2} \mu_{2}} x^{-2 \mu_{1}}=\mathcal{O}\left(x^{-2 \mu_{1}}\right), x \rightarrow+\infty .
$$

The corresponding $c d f$ will behave as

$$
F_{\mathrm{ZP}}(x)=1-\mathcal{O}\left(x^{1-2 \mu_{1}}\right), x \rightarrow+\infty .
$$


Now let

$$
\begin{aligned}
T: & F_{\mathrm{ZP}}(x) \mapsto \tilde{F}_{\mathrm{ZP}}(y)=\Phi^{-1}\left(F_{\mathrm{ZP}}\left(e^{y}\right)\right) \\
& =-\Phi^{-1}\left(\mathcal{O}\left(e^{y\left(1-2 \mu_{1}\right)}\right)\right), y \rightarrow+\infty
\end{aligned}
$$

where we have made the transformation onto lognormal paper. Now, we use (23), which applies because the argument $\mathcal{O}\left(e^{y\left(1-2 \mu_{1}\right)}\right) \rightarrow 0^{+}$as $y \rightarrow+\infty$ for $\mu_{1}>\frac{1}{2}$. We then have

$$
\tilde{F}_{\mathrm{ZP}}(y) \approx \sqrt{-2 \ln \left(2 \mathcal{O}\left(e^{y\left(1-2 \mu_{1}\right)}\right)\right)}=\mathcal{O}(\sqrt{y}), y \rightarrow+\infty .
$$

Thus the $c d f$ will behave as a square root function for high values, on lognormal paper. The limiting slope at $+\infty$ is then

$$
\lim _{y \rightarrow+\infty} \frac{\partial}{\partial y} \tilde{F}_{\mathrm{ZP}}(y)=\lim _{y \rightarrow+\infty} \frac{1}{2 \sqrt{y}}=0 .
$$

Since the limiting slope is a constant, no tail-fitting can be made at the upper tail either.

\section{REFERENCES}

[1] Y. S. Yeh and S. C. Schwartz, "Outage probability in mobile telephony due to multiple log-normal interferers"," IEEE Trans. Communications, vol. 32, pp. 380-388, Apr. 1984.

[2] M. Pratesi, F. Santucci, and F. Graziosi, "Generalized moment matching for the linear combination of lognormal RVs: application to outage analysis in wireless systems," IEEE Trans. Wireless Communications, vol. 5, pp. 1122-1132, May 2006.

[3] S. S. Szyszkowicz, "Interference in cellular networks: Sum of lognormals modeling," Master's thesis, Carleton University, Jan. 2007, can be

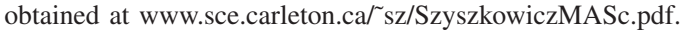

[4] S. Asmussen and L. Rojas-Nandayapa, "Asymptotics of sums of lognormal random variables with Gaussian copula," Statistics \& Probability Letters, vol. 78, pp. 2709-2714, Nov. 2008.

[5] R. B. Leipnik, "On lognormal random variables: I-the characteristic function," J. Australian Math. Soc. Ser. B, vol. 32, pp. 327-347, 1991.

[6] M. Milevsky and S. Posner, "Asian options, the sum of lognormals and the reciprocal Gamma distribution,' J. Financial and Quantitative Analysis, vol. 33, pp. 409-422, 1998.

[7] M. Romeo, V. Da Costa, and F. Bardou, "Broad distribution effects in sums of lognormal random variables," European Physical J. B, vol. 32, pp. 513-525, 2003.

[8] H. Chang and S. S. Sapatnekar, "Full-chip analysis of leakage power under process variations, including spatial correlations," ACM IEEE Design Automation Conf., pp. 523-528, 2005.

[9] L. Fenton, "The sum of log-normal probability distributions in scatter transmission systems," IEEE Trans. Communications, vol. 8, pp. 57-67, Mar. 1960.

[10] F. Berggren and S. Ben Slimane, "A simple bound on the outage probability with lognormally distributed interferers," IEEE Communications Letters, vol. 8, pp. 271-273, May 2004.

[11] C. L. J. Lam and T. Le-Ngoc, "Outage probability with correlated lognormal interferers using log shifted gamma approximation," Fifth International Conf. Information, Communications and Signal Processing (ICICS), pp. 618-622, Dec. 2005.
[12] N. B. Mehta, A. F. Molisch, J. Wu, and J. Zhang, "Approximating the sum of correlated lognormal or, lognormal-Rice random variables," IEEE ICC, vol. 4, pp. 1605-1610, June 2006.

[13] Z. Liu, J. Almhana, and R. McGorman, "Approximating lognormal sum distributions with power lognormal distributions," IEEE Trans. Vehic. Tech., vol. 57, pp. 2611-2617, July 2008.

[14] C. Tellambura, "Bounds on the distribution of a sum of correlated lognormal random variables and their application," IEEE Trans. Communications, vol. 56, pp. 1241-1248, Aug. 2008.

[15] M. Di Renzo, F. Graziosi, and F. Santucci, "Approximating the linear combination of log-normal RVs via Pearson type IV distribution for UWB performance analysis," IEEE Trans. Communications, vol. 57, pp. 388-403, Feb. 2009.

[16] S. Ben Slimane, "Bounds on the distribution of a sum of independent lognormal random variables," IEEE Trans. Communications, vol. 49, pp. 975-978, June 2001.

[17] Z. Liu, J. Almhana, F. Wang, and R. McGorman, "Mixture lognormal approximations to lognormal sum distributions," IEEE Communications Letters, vol. 11, no. 9, pp. 711-713, Sept. 2007.

[18] H. Nie and S. Chen, "Lognormal sum approximation with type IV Pearson distribution," IEEE Communications Letters, vol. 11, pp. 790792, Oct. 2007.

[19] N. Beaulieu and Q. Xie, "An optimal lognormal approximation to lognormal sum distributions," IEEE Trans. Vehicular Tech., vol. 53, pp. 479-489, Mar. 2004.

[20] J. C. S. Santos Filho, M. D. Yacoub, and P. Cardieri, "Highly accurate range-adaptive lognormal approximation to lognormal sum distributions," IEE Electronics Letters, vol. 42, pp. 361-362, Mar. 2006.

[21] Q. T. Zhang and S. H. Song, "Model selection and estimation for lognormal sums in Pearson's framework," IEEE VTC, vol. 6, pp. 28232827, May 2006.

[22] N. C. Beaulieu, A. A. Abu-Dayya, and P. J. McLane, "Estimating the distribution of a sum of independent lognormal random variables," IEEE Trans. Communications, vol. 43, pp. 2869-2873, Dec. 1995.

[23] N. C. Beaulieu and F. Rajwani, "Highly accurate simple closed-form approximations to lognormal sum distributions and densities," IEEE Communications Letters, vol. 8, pp. 709-711, Dec. 2004.

[24] L. Zhao and J. Ding, "A strict approach to approximating lognormal sum distributions," IEEE CCECE, pp. 916-919, May 2006.

[25] W. Nelson and N. Doganaksoy, "A computer program POWNOR for fitting the power-normal and -lognormal models to life or strength data from specimens of various sizes," U.S. Separtment of Commerce, National Institute of Standards and Technology, Mar. 1992.

[26] J. Kim, E. Hong, and Y. Choi, "Measurement and analysis of a massively multiplayer online role playing game traffic," in Advanced Network Conference (Network Research Workshop), Busan, Corea, Aug. 2003.

[27] S. S. Szyszkowicz and H. Yanikomeroglu, "On the tails of the distribution of the sum of lognormals," IEEE ICC, pp. 5324-5329, June 2007.

[28] A. Azzalini, "A class of distributions which includes the normal ones," Scandinavian Journal of Statistics, vol. 12, no. 2, pp. 171-178, 1985.

[29] P. Cardieri and T. S. Rappaport, "Statistics of the sum of lognormal variables in wireless communications," IEEE VTC, vol. 3, pp. 18231827, May 2000.

[30] H. E. Fettis, "A stable algorithm for computing the inverse error function in the "tail-end" region," Mathematics of Computation, vol. 28, pp. 585587, Apr. 1974. 